\title{
Influence de la teneur en caséine $\beta$ sur les caractéristiques physico-chimiques et l'aptitude à la coagulation enzymatique du lait de chèvre
}

\author{
Florent REMEUF ${ }^{\mathrm{a} *}$, Guy RICORDEAU ${ }^{\mathrm{b}}$, Ghislaine BRIGNON ${ }^{\mathrm{c}}$, \\ François GROSCLAUDE ${ }^{\mathrm{d}}$
}

\begin{abstract}
${ }^{a}$ Laboratoire de Génie et Microbiologie des Procédés Alimentaires, UMR INRA-INA Paris Grignon, 78850 Thiverval-Grignon, France

${ }^{\mathrm{b}}$ Station d'Amélioration Génétique des Animaux, INRA, Auzeville, 31326 Castanet-Tolosan Cedex, France

${ }^{\mathrm{c}}$ Unité protéines, INRA, 78352 Jouy-en-Josas Cedex, France

${ }^{\mathrm{d}}$ Laboratoire de Génétique Biochimique et de Cytogénétique, INRA, 78352 Jouy-en-Josas Cedex, France
\end{abstract}

(Reçu le 13 septembre 2000 ; accepté le 18 avril 2001)

\begin{abstract}
The influence of $\beta$-casein content on physico-chemical and renneting properties of goat's milk. Milks from goats of $\mathrm{A} / \mathrm{A}, \mathrm{A} / \mathrm{O}$ and $\mathrm{O} / \mathrm{O}$ genotypes at the $\beta$-casein locus were compared in terms of $\mathrm{N}$ fractions, micellar characteristics, Ca distribution and renneting properties. A/A milks were associated with higher casein and lower total calcium contents, lower $\mathrm{pH}$, and larger and more hydrated and mineralized micelles than $\mathrm{O} / \mathrm{O}$ milks. These showed a poor rennetability as compared with normal milks. These results were in good accordance with current micellar models, and with previous data on $\alpha_{\mathrm{s} 1}$-casein genetic polymorphism.
\end{abstract}

goat's milk / genetic polymorphism / $\beta$-casein / casein micelle / renneting property

Résumé - Les fractions protéiques et calciques de trois types de laits caprins de génotypes $\mathrm{A} / \mathrm{A}, \mathrm{A} / \mathrm{O}$ et $\mathrm{O} / \mathrm{O}$ pour la caséine $\beta$ ont été analysées. Les laits $\mathrm{A} / \mathrm{A}$ sont caractérisés par des teneurs en caséine et en protéine plus élevées, une concentration en calcium total et un $\mathrm{pH}$ plus bas, des micelles plus grosses, plus hydratées et moins minéralisées que les laits $\mathrm{O} / \mathrm{O}$. Les laits $\mathrm{O} / \mathrm{O}$ présentent une aptitude à la coagulation enzymatique très déficiente par rapport aux laits « normaux ». Ces résultats présentent

\footnotetext{
* Correspondance et tirés-à-part

Tél. : (33) 130815383 ; fax : (33) 130815597 ; e-mail : remeuf@grignon.inra.fr
} 
une bonne cohérence avec les modèles de structure micellaire actuellement admis, ainsi qu'avec les résultats déjà acquis sur les effets du polymorphisme génétique de la caséine $\alpha_{\mathrm{s} 1}$.

lait de chèvre / polymorphisme génétique / caséine $\beta$ / micelle de caséine / coagulation enzymatique

\section{INTRODUCTION}

Les effets du polymorphisme génétique des lactoprotéines sur la composition des laits et leurs propriétés technologiques ont été beaucoup étudiés chez la vache, mais dans une bien moindre mesure chez la chèvre et la brebis [11]. Chez la chèvre, ce sont surtout les effets du polymorphisme de la caséine $\alpha_{\mathrm{s} 1}$ qui ont fait l'objet de travaux récents. Différents auteurs $[3,10,12,16$, $23-25,29,35,36]$ ont montré que les allèles à fort taux de synthèse de caséine $\alpha_{\mathrm{s} 1}$ sont également associés à des concentrations en protéines et en caséines plus élevées, se traduisant par une augmentation des rendements fromagers. Les cinétiques de coagulation enzymatique montrent que les laits d'animaux homozygotes pour le variant fort $(\mathrm{A} / \mathrm{A})$ présentent une vitesse de raffermissement et une fermeté de gel finale bien supérieures à celles des laits d'animaux homozygotes pour le variant intermédiaire $(\mathrm{E} / \mathrm{E})$ et le variant faible $(\mathrm{F} / \mathrm{F})$ $[1,10,12,24,25,29]$. D'autre part, la taille moyenne des micelles est plus petite dans les laits contenant les variants à forte proportion de caséine $\alpha_{\mathrm{s} 1}$, facteur favorisant une fermeté de gel plus importante pour ces laits. En revanche, les temps de coagulation semblent moins dépendants du type de variant considéré. Selon Pierre et al. [24], les temps de gélification sont identiques pour les variants $A$ et $O$ de la caséine $\alpha_{\mathrm{s} 1}$. D'autres auteurs $[1,29]$ relèvent des temps de gélification légèrement plus élevés pour les variants à fort taux de synthèse en caséine $\alpha_{\mathrm{s} 1}$ (A) par rapport aux variants à faible taux de synthèse $(F)$. Cette différence pourrait s'expliquer par un rapport caséine $\beta$ / caséine $\alpha_{\mathrm{s} 1}$ plus grand et un degré de minéralisation micellaire plus élevé dans les laits à faible teneur en caséine $\alpha_{\mathrm{s} 1}$ [29]. Enfin, plusieurs travaux ont mis en évidence une flaveur de chèvre légèrement moins marquée dans les fromages fabriqués avec les laits de type $\alpha_{\mathrm{s} 1} \mathrm{~A} / \mathrm{A}[10,13,15,25,36]$.

Pour ce qui est de la caséine $\alpha_{\mathrm{s} 2}$, l'allèle le plus répandu est l'allèle $A$ avec une fréquence de 0,85 en races Alpine et Saanen $[4,14]$ et de 0,82 en race Pyrénéenne [31]. Cependant aucune association entre les aptitudes technologiques et les variants de cette caséine n'a été établie [2].

Concernant la caséine $\beta$, Dall'Olio et al. [9] signalent une chèvre italienne apparemment dépourvue de cette caséine, mais la première démonstration de l'existence d'un polymorphisme génétique a été apportée par Mahé et Grosclaude [17] à partir des analyses de lait de chèvres Créoles de Guadeloupe. Ce polymorphisme s'explique par la présence, en plus de l'allèle commun A, de deux « nouveaux » allèles, l'allèle $\mathrm{B}$ et un allèle nul $(\mathrm{O})$ à une fréquence voisine de 0,2 . Depuis, cet allèle a été trouvé dans la race Corse (Grosclaude et Mahé, communication personnelle), et plus particulièrement, pour ce qui concerne notre étude, dans la race Pyrénéenne, à une fréquence de 0,11 [31]. Il a également été signalé dans plusieurs races italiennes [7, 18, 27] et un deuxième allèle nul a même été caractérisé par Rando et al. [28].

Actuellement, on ne trouve pas de données publiées concernant les propriétés physico-chimiques et technologiques des laits $\mathrm{Cn}-\beta \mathrm{O}$, hormis la contribution de Chianese et al. [7]. Dans notre étude, nous avons analysé les fractions protéiques et calciques de trois types de laits caprins issus de chèvres des 3 génotypes $\mathrm{A} / \mathrm{A}, \mathrm{A} / \mathrm{O}$, $\mathrm{O} / \mathrm{O}$ pour la caséine $\beta$. Sur la base des 
modèles d'organisation micellaire actuellement admis, nous avons cherché à relier les propriétés physico-chimiques observées entre elles, ainsi qu'aux variables caractérisant les cinétiques de coagulation enzymatique de ces laits.

\section{MATÉRIEL ET MÉTHODES}

\section{1. Étude expérimentale}

\subsection{1. Échantillonnage des laits}

Les analyses ont été effectuées à partir de deux séries de prélèvements. La première, comportant 15 laits, a été réalisée le 8 août 1995 dans un seul élevage des Hautes Pyrénées, à la traite du soir, à partir d'un lot de 15 chèvres dont 5 homozygotes pour l'allèle $\beta$ nul et 10 homozygotes $\beta \mathrm{A} / \mathrm{A}$, aux environs du $5^{\mathrm{e}}$ mois de lactation. La seconde série d'échantillons a été prélevée de juillet à septembre 1998 dans 3 élevages des Hautes Pyrénées, dont un en période d'allaitement et deux en période de traite. Ces deux derniers élevages sont voisins et ont des parcours communs. Un total de 52 laits ont été prélevés dans ces trois élevages, dans le cadre d'un contrôle laitier simplifié, à partir de 7 chèvres homozygotes $\beta \mathrm{O} / \mathrm{O}$, 35 chèvres $\beta \mathrm{A} / \mathrm{A}$ et 10 chèvres hétérozygotes $\beta \mathrm{A} / \mathrm{O}$.

Sur les 15 laits de la série 1 ont été déterminés le $\mathrm{pH}$, le diamètre moyen et le degré d'hydratation des micelles, la concentration en azote non protéique, et le temps de gélification du lait emprésuré.

Pour la série 2, les 7 laits issus des chèvres $\beta \mathrm{O} / \mathrm{O}$ et 11 laits issus de chèvres $\beta \mathrm{A} / \mathrm{A}$ ont fait l'objet d'une analyse plus approfondie comportant, en plus des déterminations précédentes, la mesure des teneurs en matières azotées totales, en protéines et en caséines, celle des teneurs en calcium total et soluble, et l'appréciation du comportement à la coagulation enzymatique à l'aide du Formagraph. Pour les autres laits de la série 2, c'est-à-dire les 10 laits issus des chèvres $\beta \mathrm{A} / \mathrm{O}$ et 24 laits issus de chèvres $\beta \mathrm{A} / \mathrm{A}$, un protocole d'analyse simplifié, ne comportant ni la cinétique de coagulation, ni les déterminations des teneurs en calcium soluble et du degré d'hydratation des micelles, a été adopté.

En outre, les 67 chèvres échantillonnées dans les deux séries de prélèvements ont fait l'objet d'un typage en caséine $\alpha_{\mathrm{s} 1}$ selon la méthode électrophorétique décrite par Boulanger et al. [4] et d'un typage en caséine $\beta$ à partir de l'ADN sanguin, grâce à la méthode PCR mise au point par Persuy et al. [22].

En résumé, selon les variables considérées, et sans tenir compte des valeurs manquantes, le nombre maximal d'échantillons analysés par types de laits était de 12, 10 et 45 pour les laits issus de chèvre $\mathrm{O} / \mathrm{O}, \mathrm{A} / \mathrm{O}$ et $\mathrm{A} / \mathrm{A}$ respectivement. Ce déséquilibre du plan d'échantillonnage est lié à la structure des troupeaux (peu d'animaux porteurs de l'allèle nul), aux difficultés pratiques des prélèvements (zones de montagne) et à l'impossibilité de réaliser toutes les analyses pour tous les échantillons frais sur une période de 4 jours.

Les laits ont été prélevés juste après la traite et additionnés d'azohydrate de sodium $\left(0,4 \mathrm{~g} \cdot \mathrm{L}^{-1}\right)$ afin de pouvoir être conservés à $12{ }^{\circ} \mathrm{C}$ pendant $4 \mathrm{j}$ sans développement bactérien. Le lendemain, les laits ont été envoyés par messagerie rapide au laboratoire de Grignon pour réaliser les analyses décrites ci-dessous.

\subsubsection{Analyses physico-chimiques}

Les analyses sont réalisées sur laits écrémés par centrifugation à $600 \mathrm{~g}$ pendant $15 \mathrm{~min}$. Les teneurs en matières azotées totales, protéines, caséines, et en azote non protéique sont déterminées selon les protocoles décrits par Remeuf et al. [30].

Les teneurs en calcium soluble et total sont déterminées selon une méthode fluorimétrique à l'aide d'un appareil de type Corning calcium analyzer 940 (Corning, 
Halstead, CO9 2DX, UK). Le calcium total est dosé directement sur le lait et le calcium soluble est mesuré sur le surnageant d'ultracentrifugation à $80000 \mathrm{~g}$ pendant $70 \mathrm{~min}$ à $20^{\circ} \mathrm{C}$. Le calcium soluble est converti en $\mathrm{g} \cdot \mathrm{L}^{-1}$ de lait écrémé en multipliant la valeur obtenue sur le surnageant d'ultracentrifugation par le facteur $(1-\mathrm{x} / 1000)$, où $\mathrm{x}$ est la teneur en caséine du lait écrémé exprimée en g.L $\mathrm{L}^{-1}$. La minéralisation des micelles est calculée en rapportant la quantité de calcium colloidal $(\mathrm{Ca}$ total - Ca soluble) à la quantité de caséine.

Le diamètre moyen des micelles est mesuré par spectroscopie de corrélation photonique avec un analyseur de particule Coultronics N4 (Coulter Electronics, Miami, FL 33116-9015, États-Unis) selon le protocole décrit par Remeuf et al. [30].

L'hydratation des micelles est déterminée par mesure de l'humidité du culot d'ultracentrifugation à $80000 \mathrm{~g}$ pendant $1 \mathrm{~h}$ à $20^{\circ} \mathrm{C}[30]$.

\subsubsection{Aptitude à la coagulation par la présure}

L'aptitude à la coagulation par la présure a été appréciée sur les laits écrémés, au pH initial du lait, à l'aide du Formagraph (Foss Electric SA, Nanterre, France) selon le protocole décrit par Remeuf [29]. Trois paramètres sont déterminés: le temps de gélification (TPR), l'estimation de la fermeté du gel par la valeur de l'écartement 20 min après le temps de gélification $\left(A_{20}\right)$ et à deux fois le temps de gélification $\left(A_{2 R}\right)$. Les cinétiques de coagulation très lentes observées sur de nombreux échantillons n'ont pas permis de déterminer des valeurs fiables du paramètre $K_{20}$, ou temps mis pour obtenir un écartement de $20 \mathrm{~mm}$ des branches du Formagramme.

\subsubsection{Traitement statistique des données}

Les données recueillies dans le cadre de l'étude expérimentale ont fait l'objet d'un traitement statistique par analyse de variance à l'aide du logiciel SAS (SAS Intitute, Cary, États-Unis). Le modèle appliqué à chaque variable était le suivant :

$$
X_{i j}=\mu+\alpha_{i}+\varepsilon_{i j}
$$

$\mathrm{X}=$ variable étudiée, de moyenne générale $\mu$;

$\alpha_{\mathrm{i}}=$ effet moyen du type de lait $(1 \leq \mathrm{i} \leq 3)$;

$\varepsilon_{\mathrm{ij}}=$ erreur résiduelle $(1 \leq \mathrm{j} \leq 45)$.

\subsection{Données complémentaires}

Les résultats de l'étude expérimentale ont été complétés par deux séries de données. La première comporte l'estimation des proportions des différentes caséines réalisée sur 107 laits prélevés en juillet-août 1995 , issus de 18 chèvres $\beta \mathrm{O} / \mathrm{O}$, de 57 chèvres $\beta \mathrm{A} / \mathrm{O}$, et de 32 chèvres $\beta \mathrm{A} / \mathrm{A}$. Ces chèvres proviennent de 18 élevages choisis parmi ceux ayant participé à l'étude sur les fréquences alléliques des chèvres des Pyrénées [31], dont 15 différents de ceux utilisés pour l'étude expérimentale. Cette analyse a été effectuée par méthode HPLC [14], à l'Unité Protéines de l'INRA de Jouy-enJosas.

La seconde série de données complémentaires porte sur les taux protéiques moyens de laits contrôlés dans 7 élevages des Pyrénées Atlantiques (vallée d'Aspe) et des Hautes Pyrénées, sur 3 années (1995, 1997 et 1998), dans le cadre du contrôle laitier simplifié, avec un contrôle mensuel sur une ou deux traites et après une période d'allaitement de 2 à 3 mois.

\section{RÉSULTATS ET DISCUSSION}

Les résultats donnés dans le tableau I montrent un effet significatif du génotype de la caséine $\beta$ sur la plupart des variables mesurées. D'une manière générale, des écarts très marqués sont relevés entre les laits de type $\mathrm{A} / \mathrm{A}$ et $\mathrm{O} / \mathrm{O}$, alors qu'ils sont 
Tableau I. Effet du génotype au locus de la caséine $\beta$ sur les caractéristiques physico-chimiques et les paramètres de coagulation des laits (67 chèvres, échantillonnées en août 1995 et juillet à septembre 1998 dans 3 élevages des Pyrénées).

Table I. Effet of $\beta$-casein genotype on physicochemical and renneting properties of milks ( 67 goats, sampled in july 1995 and july to september 1998 in 3 herds of the Pyrénées area).

\begin{tabular}{|c|c|c|c|c|}
\hline \multirow[b]{2}{*}{ Variables } & \multicolumn{3}{|c|}{ Génotype au locus de la caséine $\beta^{1}$} & \multirow{2}{*}{$\begin{array}{l}\text { Signifi- } \\
\text { cation } \\
\text { de l'effe }\end{array}$} \\
\hline & $\mathrm{A} / \mathrm{A}$ & $\mathrm{A} / \mathrm{O}$ & $\mathrm{O} / \mathrm{O}$ & \\
\hline \multicolumn{5}{|l|}{ Fractions azotées } \\
\hline Matières azotées totales $\left(\mathrm{g} \cdot \mathrm{L}^{-1}\right)$ & $36,7 \pm 2,1(35)$ & $37,9 \pm 3,8(10)$ & $32,6 \pm 4,6(7)$ & NS \\
\hline Caséines totales $\left(\mathrm{g} \cdot \mathrm{L}^{-1}\right)$ & $27,9 \pm 1,9(29)$ & $26,8 \pm 3,2(10)$ & $23,5 \pm 4,5(5)$ & NS \\
\hline Caséines / MAT \% & $76,3 \pm 2,3(29)$ & $70,3 \pm 4,7(10)$ & $69,3 \pm 6,7(5)$ & $\mathrm{P}<0,01$ \\
\hline Azote non protéique $\left(\mathrm{g} \cdot \mathrm{L}^{-1}\right)$ & $0,42 \pm 0,04(39)$ & $0,44 \pm 0,08(10)$ & $0,55 \pm 0,08(12)$ & $\mathrm{P}<0,01$ \\
\hline Protéines totales $\left(\mathrm{g} \cdot \mathrm{L}^{-1}\right)$ & $34,6 \pm 2,2(29)$ & $35,2 \pm 3,7(10)$ & $28,6 \pm 4,4(7)$ & $\mathrm{P}<0,01$ \\
\hline Protéines / MAT \% & $92,4 \pm 0,84(29)$ & $92,4 \pm 1,44(10)$ & $87,8 \pm 1,73(7)$ & $\mathrm{P}<0,01$ \\
\hline \multicolumn{5}{|l|}{ pH et répartition du calcium } \\
\hline $\mathrm{pH}$ & $6,55 \pm 0,05(45)$ & $6,50 \pm 0,11(10)$ & $6,64 \pm 0,10(12)$ & $\mathrm{P}<0,05$ \\
\hline Calcium total $\left(\mathrm{g} \cdot \mathrm{L}^{-1}\right)$ & $1,23 \pm 0,08(35)$ & $1,25 \pm 0,15(10)$ & $\begin{array}{l}1,42 \pm 0,18 \\
(7)\end{array}$ & NS \\
\hline Calcium soluble $\left(\mathrm{g} \cdot \mathrm{L}^{-1}\right)$ & $0,396 \pm 0,057(12)$ & nd & $0,461 \pm 0,075(7)$ & NS \\
\hline \multicolumn{5}{|l|}{ Caractéristiques micellaires } \\
\hline Diamètre micelles (nm) & $275 \pm 13(45)$ & $265 \pm 27(10)$ & $246 \pm 25(12)$ & $\mathrm{P}<0,05$ \\
\hline Hydratation micelles $\left(\mathrm{g} \cdot \mathrm{g}^{-1}\right.$ ) & $1,74 \pm 0,07(22)$ & nd & $1,41 \pm 0,09(12)$ & $\mathrm{P}<0,01$ \\
\hline $\begin{array}{l}\text { Minéralisation des micelles } \\
\left(\mathrm{mg} \mathrm{Ca} \cdot \mathrm{g}^{-1} \text { caséine }\right)\end{array}$ & $31,6 \pm 8,8(12)$ & nd & $41,0 \pm 11(7)$ & $\mathrm{P}<0,05$ \\
\hline \multicolumn{5}{|l|}{ Paramètres de coagulation } \\
\hline Temps de gélification (min) & $15,4 \pm 8,5(21)$ & nd & $32,4 \pm 11,2(12)$ & $\mathrm{P}<0,01$ \\
\hline $\begin{array}{l}\text { Fermeté de gel à } 20 \mathrm{~min} \\
\left(\mathrm{~A}_{20}, \mathrm{~mm}\right)\end{array}$ & $20,4 \pm 5,1(11)$ & nd & $7,50 \pm 6,4(7)$ & $\mathrm{P}<0,01$ \\
\hline $\begin{array}{l}\text { Fermeté de gel à } 2 \text { fois } \\
\text { le temps de gélification } \\
\left(A_{2 R}, m m\right)\end{array}$ & $19,1 \pm 4,3(11)$ & nd & $10,0 \pm 5,4(7)$ & $\mathrm{P}<0,01$ \\
\hline $\begin{array}{l}\text { Génotype caséine } \alpha_{s 1} \\
\text { des } 67 \text { chèvres } \\
2 \text { allèles forts }(\%) \\
1 \text { allèle fort }(\%) \\
\text { aucun allèle fort }(\%)\end{array}$ & $\begin{array}{r}4 \\
23 \\
73\end{array}$ & $\begin{array}{l}30 \\
70 \\
-\end{array}$ & $\begin{array}{c}100 \\
- \\
-\end{array}$ & \\
\hline
\end{tabular}

${ }^{1}$ Pour chaque variable sont indiqués la valeur moyenne, l'intervalle de confiance à $5 \%$, et le nombre de laits analysés (entre parenthèses).

${ }^{1}$ For each variable are indicated mean value, $5 \%$ confidence interval, and number of milks analyzed (between brackets). 
plus faibles entre $\mathrm{A} / \mathrm{A}$ et $\mathrm{A} / \mathrm{O}$, ou entre $\mathrm{A} / \mathrm{O}$ et $\mathrm{O} / \mathrm{O}$, et souvent non significatifs.

\subsection{Fractions azotées}

Les laits de type A/A sont nettement plus riches en matières azotées totales (MAT), caséines et protéines que les laits de type O/O. Pour ces trois variables, les écarts moyens entre les deux types de laits s'établissent à $4,1 \mathrm{~g} \cdot \mathrm{L}^{-1}, 4,4 \mathrm{~g} \cdot \mathrm{L}^{-1}$ et $6,0 \mathrm{~g} \cdot \mathrm{L}^{-1}$ respectivement. Ceci est cohérent avec les écarts obtenus en élevages pour le taux de protéines $\left(5,1 \mathrm{~g} \cdot \mathrm{kg}^{-1}\right.$, Tab. II). Au contraire, la concentration en azote non protéique est plus élevée dans les laits contenant le variant nul. En proportion, la fraction azotée non protéique représente ainsi en moyenne environ $11,0 \%$ de l'azote total dans les laits $\mathrm{O} / \mathrm{O}$, contre $7,2 \%$ dans les laits $\mathrm{A} / \mathrm{A}$ et $7,5 \%$ dans les laits A/O. L'écart relatif plus marqué entre les laits $\mathrm{A} / \mathrm{A}$ et $\mathrm{O} / \mathrm{O}$ pour les teneurs en protéines et caséines, par rapport à ceux observés pour la MAT, se traduit par des valeurs plus faibles des ratios protéines / MAT et caséines / MAT dans les laits contenant le variant nul. Ainsi, on passe de $76,3 \%$ à $69,3 \%$ pour l'indice de caséine (caséine / MAT) entre les laits issus d'animaux homozygotes A/A et O/O. Les laits de type $\mathrm{A} / \mathrm{O}$ se situent en position intermédiaire, mais non médiane, entre les laits $\mathrm{A} / \mathrm{A}$ et $\mathrm{O} / \mathrm{O}$, étant tantôt plus proches des laits A/A (protéines, caséines, rapport protéines / MAT), tantôt plus proches des laits $\mathrm{O} / \mathrm{O}$ (rapport caséine / MAT). Il en est de même dans les contrôles en élevages, puisque la différence pondérée entre les taux de protéines des laits $\mathrm{A} / \mathrm{A}$ et $\mathrm{A} / \mathrm{O}$ est seulement de $1,0 \mathrm{~g} \cdot \mathrm{kg}^{-1}$ et peu significative (Tab. II).

L'absence de caséine $\beta$ dans les laits nuls se traduit donc par une baisse sensible de la teneur en caséine totale des laits, mais

Tableau II. Taux de protéines mesuré en élevages, dans le cadre du contrôle laitier simplifié : moyenne pondérée des différences entre génotypes caséine $\beta$, intra élevage-année ( 7 élevages sur 3 années).

Table II. Milk protein concentration measured in farms, within the framework of the simplified milk quality control: weighted average between $\beta$-casein genotypes from 7 herds sampled for 3 years.

Différence Cas A/A - Cas O/O : $\overline{\mathrm{d}}_{\mathrm{i}}=5,1 \mathrm{~g} \cdot \mathrm{kg}^{-1}(\mathrm{P}<0,01) \quad$ avec $\sum_{\mathrm{W}_{\mathrm{i}}}=14,4$

Différence Cas A/A - Cas A/O : $\overline{\mathrm{d}}_{\mathrm{i}}=1,0 \mathrm{~g} \cdot \mathrm{kg}^{-1}(\mathrm{P}=0,06) \quad$ avec $\sum_{\mathrm{W}_{\mathrm{i}}}=26,8$

Pour chaque comparaison intra élevage-année, la différence $\mathrm{d}_{\mathrm{i}}$ est pondérée par le coefficient $\mathrm{w}_{\mathrm{i}}$ qui est fonction du nombre de chèvres $\mathrm{n}_{1}$ et $\mathrm{n}_{2}$ de chaque type $\left(\mathrm{w}_{\mathrm{i}}=\frac{\mathrm{n}_{1} \times \mathrm{n}_{2}}{\mathrm{n}_{1}+\mathrm{n}_{2}}\right)$. La différence moyenne $\mathrm{d}_{\mathrm{i}}$ a pour expression $: \overline{\mathrm{d}}_{\mathrm{i}}=\frac{\sum \mathrm{w}_{\mathrm{i}} \mathrm{d}_{\mathrm{i}}}{\sum \mathrm{w}_{\mathrm{i}}}$

Difference Cas A/A - Cas O/O : $\overline{\mathrm{d}}_{\mathrm{i}}=5.1 \mathrm{~g} \cdot \mathrm{kg}^{-1}(\mathrm{P}<0.01) \quad$ with $\sum_{\mathrm{W}_{\mathrm{i}}}=14.4$ Difference Cas A/A - Cas A/O : $\overline{\mathrm{d}}_{\mathrm{i}}=1.0 \mathrm{~g} \cdot \mathrm{kg}^{-1}(\mathrm{P}=0.06) \quad$ with $\sum_{\mathrm{W}_{\mathrm{i}}}=26.8$

For each comparison within herd-year, the difference $d_{i}$ is wheighted with coefficient $w_{i}$ wich is a function of the number of goats $n_{1}$ and $n_{2}$ of each type $\left(w_{i}=\frac{n_{1} \times n_{2}}{n_{1}+n_{2}}\right)$ The average difference $\mathrm{d}_{\mathrm{i}}$ is expressed as $\overline{\mathrm{d}}_{\mathrm{i}}=\frac{\sum \mathrm{w}_{\mathrm{i}} \mathrm{d}_{\mathrm{i}}}{\sum \mathrm{w}_{\mathrm{i}}}$. 
beaucoup moins qu'on ne pouvait le supposer, sachant que la caséine $\beta$ représente de $55 \%$ à $57 \%$ de la caséine totale dans un lait normal [30] (cf. Tab. III). Il semble donc que la situation ne soit pas comparable à celle qui prévaut dans le cas du polymorphisme de la caséine $\alpha_{s 1}$, pour lequel il n'y a pas compensation, ou très peu (Grosclaude et Manfredi, résultats non publiés). Dans le cas de la comparaison des laits de type $\alpha_{\mathrm{s} 1}$ A, E et F, l'écart observé entre les taux de synthèse des caséines correspond approximativement à celui observé entre les taux de caséines totales. Dans le cas du polymorphisme de la caséine $\beta$, le phénomène de compensation pourrait s'expliquer par plusieurs mécanismes, dont le premier est l'existence d'une association (haplotype) entre l'allèle nul en caséine $\beta$ et l'allèle fort B4 en caséine $\alpha_{\mathrm{s} 1}$ (Grosclaude, résultats non publiés). L'existence de cet haplotype $\alpha_{\mathrm{s} 1}$ B4- $\beta$ O est effectivement confirmée par l'examen des génotypes caséine $\alpha_{\mathrm{s} 1}$ des 67 chèvres de l'étude expérimentale (Tab. I) et des 107 chèvres échantillonnées en 1995 dans 18 élevages des Pyrénées (Tab. III, [31]). Ainsi, les homozygotes $\beta$ nuls sont toujours homozygotes $\alpha_{\mathrm{s} 1} \mathrm{~B} 4$, et les hétérozygotes $\beta \mathrm{A} / \mathrm{O}$ sont au moins porteurs de l'allèle fort $\alpha_{\mathrm{s} 1} \mathrm{~B} 4$. En revanche, les chèvres $\beta$ A/A comptent de 23 à $47 \%$ d'individus portant un allèle fort (Tabs. I et III) et très peu d'homozygotes allèles forts, $4 \%$ seulement dans la série des 67 chèvres de l'étude expérimentale. L'existence de l'haplotype $\left(\alpha_{\mathrm{s} 1} \mathrm{~B} 4-\beta \mathrm{O}\right)$ ne permet donc pas de comparer les génotypes en caséine $\beta$ à même teneur en caséine $\alpha_{\mathrm{s} 1}$, puisque les chèvres homozygotes et hétérozygotes $\beta$ nulles ont, en moyenne, un taux de caséine $\alpha_{\mathrm{s} 1}$ plus élevé que les chèvres normales (A/A). Dans l'ensemble, la réduction du pourcentage de caséine $\beta$ des laits $\mathrm{A} / \mathrm{A}$ à $\mathrm{A} / \mathrm{O}$ puis $\mathrm{O} / \mathrm{O}$, se traduit essentiellement par une augmentation du pourcentage de caséine $\alpha_{\mathrm{s} 1}$, de $12 \%$ à $23 \%$ puis $47 \%$ respectivement (Tab. III). A cet effet dû à l'haplotype, pourrait s'ajouter un mécanisme physiologique décrit par Chanat et al. [6]. Selon ces auteurs, chez la chèvre, la synthèse protéique dans la cellule mammaire serait ralentie pour les chèvres pourvues d'allèles déficients en caséine $\alpha_{\mathrm{s} 1}$. La présence de caséine $\alpha_{\mathrm{s} 1}$ favoriserait au contraire le transport de l'ensemble des caséines du réticulum endoplasmique vers l'appareil de Golgi. Ce mécanisme pourrait avoir un effet compensateur partiel sur la synthèse de caséines dans le cas des animaux pourvus de l'haplotype $\alpha_{\mathrm{s} 1}$ B4- $\beta$ O. Enfin, il est possible aussi que le phénomène de compensation soit dû pour partie à la synthèse de formes protéiques tronquées (peptides), consécutives à la mutation nonsens qui est à l'origine du phénotype $\beta$ nul [22]. Pour les laits issus d'animaux hétéro-

Tableau III. Proportions des différentes caséines en fonction du génotype au locus de la caséine $\beta$ (107 chèvres, échantillonnées en juillet et août 1995 dans 18 élevages des Pyrénées).

Table III. Proportions of caseins as influenced by $\beta$-casein genotype (107 goats, sampled in july and august 1995 in 18 herds of the Pyrénées area).

\begin{tabular}{|c|c|c|c|c|c|c|c|c|}
\hline \multirow{2}{*}{$\begin{array}{c}\text { Génotype } \\
\text { au locus } \\
\text { de la } \\
\text { caséine } \beta\end{array}$} & \multicolumn{4}{|c|}{$\begin{array}{c}\text { Pombre } \\
\text { de laits }\end{array}$} & \multicolumn{3}{|c|}{$\begin{array}{c}\text { Génotype au locus } \\
\text { de la caséine } \alpha_{\mathrm{s} 1}\end{array}$} \\
\cline { 2 - 6 } & $\begin{array}{c}\text { Caséine } \\
\alpha_{\mathrm{s} 1}\end{array}$ & $\begin{array}{c}\text { Caséine } \\
\alpha_{\mathrm{s} 2}\end{array}$ & $\begin{array}{c}\text { Caséine } \\
\beta\end{array}$ & $\begin{array}{c}\text { Caséine } \\
\kappa\end{array}$ & & $\begin{array}{c}2 \text { allèles } \\
\text { forts }\end{array}$ & $\begin{array}{c}1 \text { allèle } \\
\text { fort }\end{array}$ & $\begin{array}{c}0 \text { allèle } \\
\text { fort }\end{array}$ \\
\hline $\mathrm{A} / \mathrm{A}$ & 12,1 & 15,7 & 57,0 & 14,5 & 32 & 0 & 47 & 53 \\
\hline $\mathrm{A} / \mathrm{O}$ & 23,1 & 18,4 & 39,1 & 17,4 & 57 & 26 & 74 & - \\
\hline $\mathrm{O} / \mathrm{O}$ & 47,3 & 22,3 & - & 25,8 & 18 & 100 & - & - \\
\hline
\end{tabular}


zygotes, le principe de co-dominance entre allèles devrait se traduire par des valeurs médianes entre $\mathrm{O} / \mathrm{O}$ et $\mathrm{A} / \mathrm{A}$ pour les variables quantitatives. La situation différente observée pourrait être liée au déséquilibre de l'échantillonnage, mais également à l'existence de l'haplotype $\alpha_{\mathrm{s} 1} \mathrm{~B} 4-\beta \mathrm{O}$, ou aux mécanismes de régulation de la synthèse de caséine décrits précédemment. Enfin, bien qu'aucune donnée ne permette d'étayer cette hypothèse actuellement, la teneur plus élevée en azote non protéique dans les laits $\mathrm{O} / \mathrm{O}$ pourraient être l'indicateur d'une synthèse protéique moins efficace dans les laits de type $\mathrm{O} / \mathrm{O}$. Elle est également compatible avec le mécanisme de synthèse de formes protéiques tronquées évoqué précédemment.

\section{2. $\mathrm{pH}$ et répartition du calcium}

Le $\mathrm{pH}$ des laits $\mathrm{O} / \mathrm{O}$ est légèrement supérieur à celui des laits $\mathrm{A} / \mathrm{A}$ et $\mathrm{A} / \mathrm{O}$. Les laits $\mathrm{O} / \mathrm{O}$ sont également caractérisés par une concentration moyenne en calcium total plus élevée que les laits $\mathrm{A} / \mathrm{A}$. Un écart allant dans le même sens est observé pour la teneur en calcium soluble. En revanche, la répartition $\mathrm{du}$ calcium entre les phases soluble et colloïdale apparaît identique pour les deux types de laits, avec 32,2\% de calcium soluble dans les laits $\mathrm{A} / \mathrm{A}$ et $32,5 \%$ dans les laits nuls. On sait que les caséines contribuent, avec les phosphates et les citrates, à fixer le $\mathrm{pH}$ du lait, qui est d'autant plus bas que la teneur en caséine est élevée [20]. Il est donc logique de trouver associées des valeurs de $\mathrm{pH}$ basses et des teneurs en caséines élevées dans les laits issus d'animaux homozygotes $\mathrm{A} / \mathrm{A}$ et hétérozygotes $\mathrm{A} / \mathrm{O}$. La plus grande richesse des laits $\mathrm{O} / \mathrm{O}$ en calcium pourrait s'expliquer par le fait que ces laits contiennent relativement plus de caséines $\alpha_{\mathrm{s} 1}$ et $\alpha_{\mathrm{s} 2}$ (Tab. III). On sait en effet que les caséines $\alpha_{\mathrm{s}}$ présentent une capacité de fixation du calcium supérieure à celle de la ca - séine $\beta$ du fait d'un plus grand nombre de groupements phosphates [5].

\subsection{Caractéristiques micellaires}

Les laits $\mathrm{A} / \mathrm{A}$ et $\mathrm{O} / \mathrm{O}$ diffèrent très nettement par plusieurs caractéristiques micellaires importantes. Le diamètre moyen des micelles est significativement plus élevé dans les laits $\mathrm{A} / \mathrm{A}$ que dans les laits $\mathrm{O} / \mathrm{O}$ : $275 \mathrm{~nm}$ contre $246 \mathrm{~nm}$. Les laits d'animaux hétérozygotes présentent des dimensions micellaires médianes. L'examen des proportions de caséines (Tab. III) permet de retrouver la corrélation négative classique entre le pourcentage de caséine $\kappa$ et la taille des micelles, due à la localisation préférentiellement périphérique de cette caséine au sein de l'édifice micellaire.

La teneur en caséine $\beta$ influence également très significativement le degré d'hydratation des micelles. Ce paramètre présente, dans les laits $\mathrm{A} / \mathrm{A}$, des valeurs nettement supérieures $\left(1,74 \mathrm{~g} \cdot \mathrm{g}^{-1}\right)$ à celles trouvées dans les laits $\mathrm{O} / \mathrm{O}\left(1,41 \mathrm{~g} \cdot \mathrm{g}^{-1}\right)$. Dans le même temps, la minéralisation moyenne des micelles est beaucoup plus élevée dans les laits de type $\mathrm{O} / \mathrm{O}$ : $41 \mathrm{mg} \mathrm{Ca} \cdot \mathrm{g}^{-1}$ caséine contre $31,6 \mathrm{mg} \mathrm{Ca} \cdot \mathrm{g}^{-1}$ caséine dans les laits de type A/A. Cet écart important est probablement imputable à la substitution de la caséine $\beta$ par les caséines $\alpha_{\mathrm{s} 1}$ et $\alpha_{\mathrm{s} 2}$ (Tab. III) qui présentent une plus forte capacité de fixation du calcium.

Il a été établi que la quantité de calcium micellaire est corrélée négativement à l'hydratation des micelles [33], puisque les molécules d'eau et le calcium sont susceptibles de se fixer tous les deux sur les groupements chargés des acides aminés polaires des caséines [8]. Cette relation minéralisation - solvatation a été confirmée pour les micelles caprines par Remeuf et al. [30]. Il est donc cohérent d'observer dans les laits $\mathrm{O} / \mathrm{O}$ des micelles à la fois plus minéralisées et moins hydratées que dans les laits A/A. Une telle différence de solvatation des micelles n'avait pas été notée pour des laits 
contenant des variants différents de la caséine $\alpha_{\mathrm{s} 1}$ [29]. Mais d'une part, l'écart de minéralisation calcique des micelles entre des laits à teneurs faible et élevée en caséine $\alpha_{\mathrm{s} 1}$ est plus limité que celle observée ici, et d'autre part, la dimension des micelles joue probablement un effet compensateur plus marqué. En effet, la diminution de taille micellaire importante observée dans les laits riches en caséine $\alpha_{\mathrm{s} 1}$, par rapport aux laits pauvres (écart moyen de $47 \mathrm{~nm}$ [29]), engendre mécaniquement une augmentation de l'hydratation par accroissement du rapport surface/volume des micelles.

Chez la vache, mais aussi chez la chèvre, il est admis que la caséine $\kappa$ est située principalement à la surface des micelles et a un effet stabilisateur sur la suspension micellaire. En revanche, la répartition et le rôle structurant des trois autres caséines restent encore discutés [5]. Les données expérimentales présentées ici semblent indiquer cependant que les laits caprins sans ca séine $\beta$ sont caractérisés par une structure micellaire plus dense, plus compacte, se traduisant par des micelles plus petites et moins hydratées. La présence des caséines $\alpha_{\mathrm{s} 1}$ et $\alpha_{\mathrm{s} 2}$ en proportion élevée aurait ainsi tendance à « resserrer » l'organisation supra-moléculaire de la micelle, grâce à leur forte capacité à établir des ponts salins inter-moléculaires. Martin et al. [19] suggèrent que la caséine $\alpha_{\mathrm{s} 1}$ joue un rôle déterminant dans le transport intracellulaire des caséines et la formation des micelles. Cette hypothèse est étayée par les observations récentes de Tziboula et Horne [35], selon lesquelles le variant $\mathrm{F}$ de la caséine $\alpha_{\mathrm{s} 1}$, dépourvu d'une partie des sites de phosphorylation du variant d'origine, est associé à une répartition plus périphérique de la caséine au sein de la micelle. Dans les laits correspondants, les micelles sont plus grosses [23, $26,29]$, ce qui pourrait indiquer une structure moins compacte, plus relâchée, dans laquelle le variant $\mathrm{F}$ de la caséine $\alpha_{\mathrm{s} 1}$, moins phosphorylé, n'aurait pas le même effet de pontage entre les molécules caséiques. Les observations réalisées dans le présent travail, à partir des laits de chèvres pyrénéennes, vont dans le même sens, puisque les micelles dépourvues de caséine $\beta$ sont proportionnellement plus riches en caséines $\alpha_{\mathrm{s}}\left(\alpha_{\mathrm{s} 1}\right.$ et $\left.\alpha_{\mathrm{s} 2}\right)$, et présentent une taille réduite par rapport aux micelles des laits A/A.

\subsection{Aptitude à la coagulation}

Il existe une différence très significative de comportement à la coagulation enzymatique entre les laits $\mathrm{O} / \mathrm{O}$ et $\mathrm{A} / \mathrm{A}$. Les laits $\mathrm{O} / \mathrm{O}$ présentent un temps de gélification pratiquement double de celui des laits A/A. Par ailleurs, la cinétique de raffermissement du gel est également fortement influencée par le type génétique. Les valeurs de fermeté à $20 \mathrm{~min}$, et à 2 fois le temps de gélification, sont beaucoup plus faibles dans les laits $\mathrm{O} / \mathrm{O}$, traduisant une capacité à la réticulation et au raffermissement très réduite dans ces laits. Ces observations sont concordantes avec celles réalisées par Chianese et al. [7] qui indiquent des temps de gélification plus longs (15 à $25 \mathrm{~min}$ contre 4,5 à $7 \mathrm{~min}$ ), et des valeurs de $\mathrm{K}_{20}$ et $\mathrm{A}_{30}$ non mesurables, pour les laits dépourvus de caséine $\beta$ par rapport à des laits normaux. La moindre aptitude des laits $\mathrm{O} / \mathrm{O}$ à la coagulation enzymatique peut s'expliquer par leurs caractéristiques physico-chimiques particulières. Il est clair que, par rapport aux laits $\mathrm{A} / \mathrm{A}$, le $\mathrm{pH}$ moyen plus élevé de ces laits, et la teneur en caséine plus faible, constituent des facteurs défavorables essentiels pour le comportement de ces laits vis-à-vis de la présure. Il a été montré, en particulier chez la chèvre, qu'une augmentation de la proportion de caséines $\alpha_{\mathrm{s}} \mathrm{s}$ 'accompagne d'un allongement du temps de gélification [30]. En revanche, l'hydratation faible et la minéralisation élevée des micelles devraient contribuer à améliorer l'aptitude à la coagulation enzymatique des laits $\beta$ nuls, en favorisant les phases d'agrégation et de raffermissement. Les dimensions micellaires plus faibles observées 
dans les laits issus d'animaux homozygotes $\mathrm{O} / \mathrm{O}$ sont également propices à l'obtention d'un gel plus ferme $[21,30]$. Les laits $\mathrm{O} / \mathrm{O}$ sont donc caractérisés par un ensemble de propriétés physico-chimiques dont certaines ont un effet positif, et d'autres un effet négatif sur le processus de coagulation par voie enzymatique. Dans cet équilibre, il semble que le $\mathrm{pH}$ et la teneur en caséine totale, qui sont les deux paramètres majeurs de l'aptitude des laits à la coagulation enzymatique, constituent les facteurs limitants. Il serait intéressant à ce sujet de compléter ces données par une étude comparative des comportements à la coagulation de laits $\mathrm{A} / \mathrm{A}$ et $\mathrm{O} / \mathrm{O}$ dont les $\mathrm{pH}$ et les teneurs en caséines seraient ramenées artificiellement à des niveaux équivalents.

\section{CONCLUSION}

Ces résultats montrent un effet quantitatif très marqué du polymorphisme de la caséine $\beta$ sur les caractéristiques physicochimiques et le comportement à la coagulation enzymatique des laits. Les laits dépourvus de caséine $\beta$ présentent, en comparaison avec des laits «normaux», une teneur en caséine plus faible, et une aptitude à la coagulation enzymatique fortement dégradée. Il s'agit d'une situation assez similaire à celle rencontrée avec les laits à faible teneur, ou dépourvus, de caséine $\alpha_{\mathrm{s} 1}$. Les relations entre les différents paramètres physico-chimiques mesurés sont cohérentes avec les modèles micellaires actuellement connus, et avec les résultats obtenus antérieurement sur des laits se distinguant par le type génétique de ca séine $\alpha_{\mathrm{s} 1}$. Cette étude souligne aussi l'intérêt, au plan fondamental, de travaux comparatifs réalisés à partir de systèmes micellaires natifs dont les caractéristiques diffèrent nettement. Elle pourrait être complétée par d'autres travaux à venir visant par exemple à comparer les caractéristiques des laits selon le génotype de caséine $\beta$, à teneurs en caséine égales et en tenant compte en parallèle du polymorphisme des caséines $\alpha_{\mathrm{s} 1}$ et $\alpha_{\mathrm{s} 2}$. Il serait également intéressant d'étendre ces recherches à d'autres aspects de la stabilité colloïdale, en particulier la stabilité thermique [34]. Le rôle essentiel de la caséine $\kappa$ dans l'assemblage et la stabilité des micelles [32] laisse à penser que l'enrichissement en caséine $\kappa$ constaté dans les laits $\beta \mathrm{O} / \mathrm{O}$ pourrait conduire à des micelles de taille réduite (ce qui est observé dans cette étude) et plus stables vis-à-vis des traitements thermiques.

\section{REMERCIEMENTS}

Nous remercions M.A. Persuy et M.F. Mahé (INRA, Laboratoire de Génétique biochimique et de Cytogénétique à Jouy-en-Josas), qui ont contribué respectivement aux typages des caséines $\beta$ et $\alpha_{\mathrm{s}}$, ainsi que A. Audiot (Conservatoire du patrimoine biologique de MidiPyrénées, INRA-Auzeville) et P. Barbot (Syndicat caprin inter-départemental, 31390 Carbonne) qui ont permis les prélèvements de lait en élevages dans le cadre d'un contrôle laitier simplifié.

\section{RÉFÉRENCES}

[1] Ambrosoli R., Di Stasio L., Mazzocco P., Content of $\alpha_{\mathrm{s} 1}$-casein and coagulation properties in goat milk, J. Dairy Sci. 71 (1988) 24-28.

[2] Barbieri M.E., Polymorphisme de la caséine $\alpha_{\mathrm{s} 1}$ Effets des génotypes sur les performances zootechniques et utilisation en sélection caprine. Thèse INA Paris-Grignon, 1995, 161 p.

[3] Barbieri M.E., Manfredi E., Elsen J.M., Ricordeau G., Bouillon J., Grosclaude F., Mahé M.F., Bibé B., Effet du locus de la caséine $\alpha_{\mathrm{s} 1}$ sur les performances laitières et les paramètres génétiques des chèvres Alpine, Genet. Sel. Evol. 27 (1995) 437-450.

[4] Boulanger A., Grosclaude F., Mahé M.-F., Polymorphisme des caséines $\alpha_{\mathrm{s} 1}$ et $\alpha_{\mathrm{s} 2}$ de la chèvre (Capra hircus), Genet. Sel. Evol. 16 (1984) 157-176.

[5] Brulé G., Lenoir J., Remeuf F., La micelle de caséine et la coagulation du lait, in : Eck A., Gillis J.C. (Eds.), Le Fromage, Lavoisier Tec \& Doc, Paris, 1997, pp. 7-41.

[6] Chanat E., Martin P., Ollivier-Bousquet M., $\alpha_{\mathrm{s} 1}{ }^{-}$ casein is required for the efficient transport of $\beta$ - 
and $\kappa$-casein from the endoplasmic reticulum to the Golgi apparatus of mammary epithelial cells. J. Cell Sci. 112 (1999) 3399-3412.

[7] Chianese L., Garro G., Nicolai M.A., Mauriello R., Ferranti P., Pizzano R., Cappuccio U., Laezza P., Addeo F., Ramunno L., Rando A., Rubino R., The nature of $\beta$-casein heterogeneity in caprine milk, Lait 73 (1993) 533-547.

[8] Dalgleish D.G., Parker T.G., Binding of calcium ions to bovine $\alpha_{\mathrm{s} 1}$-casein and precipitation of the protein-calcium ion complexes, J. Dairy Res. 47 (1980) 113-122.

[9] Dall'Olio S., Davoli R., Russo V., Una nuova variante di caseina caprina. Sci. Tec. LattCasearia 40 (1989) 24-28.

[10] Delacroix-Buchet A., Degas C., Lamberet G., Vassal L., Influence des variants AA et FF de la caséine $\alpha_{\mathrm{s} 1}$ caprine sur le rendement fromager et les caractéristiques sensorielles des fromages, Lait 76 (1996) 217-241.

[11] F.I.L.-I.D.F. Milk protein polymorphism. Special issue 9702, Int. Dairy Fed. Brussels, Belgium, 1997, $480 \mathrm{p}$.

[12] Grosclaude F., Ricordeau G., Martin P., Remeuf F., Vassal L., Bouillon J., Du gène au fromage : le polymorphisme de la caséine $\alpha_{\mathrm{s} 1}$ caprine, ses effets, son évolution, INRA Prod. Anim. 7 (1994) 3-19.

[13] Heil F., Dumont J.P., Caractéristiques organoleptiques de fromages de chèvres fabriqués à partir de laits contenant des variants génétiques différents de la caséine $\alpha_{\mathrm{s} 1}$, Lait 73(1993) 559-565.

[14] Jaubert A., Martin P., Reverse-phase HPLC analysis of goat caseins. Identification of $\alpha_{\mathrm{s} 1}$ and $\alpha_{\mathrm{s} 2}$ genetic variants, Lait 72 (1992) 235-247.

[15] Lamberet G., Degas C., Delacroix-Buchet A., Vassal L., Influence de caractères liés aux allèles A et $F$ de la caséine $\alpha_{\mathrm{s} 1}$ caprine sur la flaveur chèvre : fabrications fromagères avec échange de protéines et de matières grasses, Lait 76 (1996) 349-361.

[16] Mahaut M., Korolczuk J., Effect of genetic variants of casein on the recovery of milk proteins in fresh and soft cheeses obtained by ultra- or microfiltration from goat's milk, in : Cheese yield and factors affecting its control. Special issue 9402, Int. Dairy Fed., Brussels, Belgium 1993, 174-178.

[17] Mahé M.F., Grosclaude F., Polymorphism of $\beta$ casein in the Creole goat of Guadeloupe : evidence for a null allele, Genet. Sel. Evol. 25 (1993) 403-408.

[18] Marletta D., Bordonaro S., Guastella A.M., Avondo M., D'Urso G., Genomic analysis of the $\alpha_{\mathrm{s} 1}$ and $\beta$ casein loci in "Girgentana » and « Argentata dell'Etna » goats, in : Proceed. 7 e Conf. Intern. sur les caprins, Tours, France, 2000, 724.

[19] Martin P., Ollivier-Bousquet M. Grosclaude F., Genetic polymorphism of caseins: a tool to in- vestigate casein micelle organization, Int. Dairy J. 9 (1999) 163-171.

[20] Mathieu J., Initiation à la physicochimie du lait, Lavoisier Tec et Doc, Paris, 1998, 220 p.

[21] Niki R., Arima S., Effects of size of casein micelle on firmness of rennet curd, Jpn. J. Zootech. Sci. 55 (1984) 409-415.

[22] Persuy M.A., Printz C., Medrano J.F., Mercier J.C., A single nucleotide deletion resulting in a premature stop codon is associated with marked reduction of transcripts from a goat $\beta$-casein null allele, Ann. Genet. 30 (1999) 444-451.

[23] Pierre A., Michel F., Le Graët Y., Variation in size of goat milk casein micelles related to casein genotype, Lait 75 (1995) 489-502.

[24] Pierre A., Le Quéré L., Riaublanc A., Le Graët Y., Demaizières D., Michel F., Composition and physicochemical characteristics of goat milks containing the $\mathrm{A}$ or $\mathrm{O} \alpha_{\mathrm{s} 1}$ casein variants, Lait 78 (1998) 191-202.

[25] Pierre A., Le Quéré L., Famelart M.H., Riaublanc A., Rousseau F., Composition, yield, texture and aroma compounds of goat cheeses as related to the $\mathrm{A}$ and $\mathrm{O}$ variants of $\alpha_{\mathrm{s} 1}$ casein in milk, Lait 78 (1998) 291-301.

[26] Pierre A., Michel F., Le Graët Y., Zahoute L., Casein micelle size in relation with casein composition and $\alpha_{\mathrm{s} 1}, \alpha_{\mathrm{s} 2}, \beta$ and $\kappa$ casein contents in goat milk, Lait 78 (1998) 591-605.

[27] Ramunno L., Longobardi E., Cosenza G., Pastore N., Gallo D., Di Berardino D., Rubino R., Calandrelli M., Rando A., Genetic structure of the $\alpha_{\mathrm{s} 1}, \beta$ and $\alpha_{\mathrm{s} 2}$ casein loci of a goat population reared in the province of Naples, in : Proceed. 7 e Conf. Intern. sur les caprins, Tours, France, 2000, 725.

[28] Rando A., Pappalardo M., Capuano M., Di Gregorio P., Ramunno L., Two mutations might be responsible for the absence of $\beta$-casein in goat milk, in : Proceed. 25th Int. Society of Animal Genetics Conference, Tours, France, 1996, 31.

[29] Remeuf F., Influence du polymorphisme génétique de la caséine $\alpha_{\mathrm{s} 1}$ caprine sur les caractéristiques physico-chimiques et technologiques du lait, Lait 73 (1993) 549-557.

[30] RemeufF., Lenoir J., Duby C., Étude des relations entre les caractéristiques physico-chimiques des laits de chèvre et leur aptitude à la coagulation par la présure, Lait 69 (1989) 499-518.

[31] Ricordeau G., Mahé M.F., Persuy M.A., Leroux C., François V., Amigues Y., Fréquences alléliques des caséines chez les chèvres des Pyrénées. Cas particulier de la caséine $\beta$ nulle, INRA Prod. Anim. 12 (1999) 29-38.

[32] Rollema H.S., Casein association and micelle formation, in : P.F. Fox (Ed.), Advanced dairy chemistry. Vol 1. Proteins, Elsevier Applied Science Publishers, London, 1992, pp 111-140. 
[33] Sood S.M., Gaind D.K., Dewan R.K., Correlation between micelle solvation and calcium content, N. Z. J. Dairy Sci. 14 (1979) 32-34.

[34] Tziboula A., Casein diversity in caprine milk and its relation to technological properties: heat stability, Int. J. Dairy Technol. 50 (1997) 134-138.

[35] Tziboula A., Horne D.S., The role of $\alpha_{\mathrm{s} 1}$-casein in the structure of caprine casein micelles, Int. Dairy J. 9 (1999) 173-178.
[36] Vassal L., Delacroix-Buchet A., Bouillon J., Influence des variants AA, EE et FF de la caséine $\alpha_{\mathrm{s} 1}$ caprine sur le rendement fromager et les caractéristiques sensorielles de fromages traditionnels : premières observations, Lait 74 (1994) 89-103.

To access this journal online: www.edpsciences.org 